\title{
Investigation of the size distribution for diffusion-controlled drug release from Drug Delivery Systems of various geometries
}

\author{
T.I. Spiridonova ${ }^{a}$, S.I. Tverdokhlebova, Y.G. Anissimov, \\ a Tomsk Polytechnic University, 30 Lenin Avenue, Tomsk 634050, Russian Federation \\ ${ }^{\mathrm{b}}$ School of Environment and Sciences, Griffith University, Gold Coast, Queensland, 4222, Australia \\ ${ }^{\mathrm{c}}$ Institute of Molecular Medicine, Sechenov First Moscow State Medical University, Moscow, Russia \\ * Corresponding author. \\ E-mail address: y.anissimov@griffith.edu.au (Y.G. Anissimov).
}

Keywords: Mathematical modeling, Diffusion, Drug Delivery Systems, Controlled drug release, Nanoparticles, Microparticles

\begin{abstract}
Various Drug Delivery Systems (DDSs) are often used in modern medicine to achieve controlled and targeted drug release. Diffusional release of drugs from DDSs is often the main mechanism, especially at early times. Generally, average dimensions of DDS are used to model the drug release, but our recent work on drug release from fibers demonstrated that taking into account diameter distribution is essential. This work systematically investigated the effect of size distribution on diffusional drug release from DDSs of various geometric forms such as membranes, fibers and spherical particles. The investigation clearly demonstrated that the size distribution has the largest effect on the drug release profiles from spherical particles compared to other geometric forms. Published experimental data for drug release from polymer micro- and nanoparticles were fitted and the diffusion coefficients were determined assuming reported radius distributions. Assuming the average radius when fitting the data leads to up to 5 times underestimation of the diffusion coefficient of drug in the polymer.
\end{abstract}

\section{Introduction}

In modern medicine there is a trend towards targeted local drug delivery. This approach allows to reduce the toxic effect of drugs on various organs and increase their effectiveness at a required site of treatment. One of the most promising materials for the fabrication of targeted drug delivery systems (DDSs) are polymers [1], [2]. Due to their good biocompatibility and physical and chemical properties, polymers are widely used in various fields of medicine, including as agents for targeted delivery of drugs, growth factors, DNA molecules and living cells [3]. DDSs of various geometries such as membranes [4], [5], fibers [6], [7], micro- and nanoparticles [8]-[12] have been previously developed.

Polymer DDSs can be prepared by a variety of methods such as dispersion of preformed polymers, solvent evaporation method, polymerization method, electrospinning method etc. [11]. The influence of the shape and size of the device on the drug release is significant and its mathematical modeling has been previously considered [13]-[16]. Precise mathematical modeling of drug release is essential for development of DDSs with tailored drug release profiles [15]-[18].

Often it is not practical or necessary to produce DDSs with identical dimensions. As a result, DDSs consisting of elements of different sizes are obtained, with some distribution in their dimensions. Such distributions in dimensions were previously reported in many experimental works [19]-[25] and could influence drug release profiles from such DDSs.

Some existing mathematical models also take into account the size distribution of particles in an ensemble. One of the first theoretical papers dedicated to this topic was work by Dappert et al. [26], in which the model for single particle has been incorporated into a statistical model for the ensemble of microcapsules. Later it was continued by Gross et al. [27], Donbrow et al. [28] and 
Benita. Then Grassi et al. [29] included into consideration other factors besides the particle size distribution, such as physical state of the drug inside the polymeric particles, the viscoelastic properties of the polymer-penetrant system and the dissolution-diffusion properties of the system. A mathematical approach to splitting an ensemble of particles into groups similar to that used in this work was developed in article [30]. In this work, it was also considered that each group of particles makes an independent contribution to the release and presents the individual release characteristics for each group. A more detailed literature review can be found in the article of Kaunisto et al. [31]. It is also necessary to note the work of recent years, in particular, articles [32] and [33]. The first article mostly modeled the melting enthalpy/temperature variation and the solubility dependence on nanocrystals radius, but the release process from an ensemble of polymeric particles (crosslinked polyvinylpyrrolidone) was also considered. While most models of drug release from polydisperse systems were empirical, in the article [33] a new mechanistic model for describing the single pellet as well as mono- and poly-disperse ensembles of pellets behavior in terms of hydration, drug dissolution, diffusion and particles size distribution was offered. All particles were divided into several groups with an average number for each group, similarly to this work. In addition, it should be noted that the authors of [33] provided the MATLAB program code for the implementation of their model. That model [33] was subsequently successfully applied in practice in work [34] for investigation of effect of tetrodotoxin pellets in a rat model of postherpetic neuralgia. These mathematical models described the drug release from an ensemble of coated pellets and capsules, with the release described by empirical functions or by ordinary differential equations. The present work considers the polymer systems with uniformly distributed drug, with the diffusional release described by partial differential equations, taking into account the particle size distribution. Such systems are common when polymer DDSs are produced by the method of electrospinning, one of the most used method. In this case the drug is usually distributed evenly throughout the volume of the polymer matrix [35].

We have previously proposed the mathematical approach that allows to take into account membrane thickness [36] and fibers radius [37] distributions for accurate prediction of drug release from flat and cylindrical DDSs. The influence of various forms of fiber radius distribution on drug release from the DDSs were investigated [37]. It was clearly demonstrated that accounting for a wide radius distribution significantly increases the accuracy of drug release predictions and diffusion coefficient determination from fitting experimental data. It was also shown that the relative errors in determining diffusion coefficient using the model with radius fixed to the mean value stays under $10 \%$ only for scaffolds with relatively narrow fiber radius distribution (with coefficient of variation, $C V$, less than 0.2 ). At the same time, in practice, wider fiber distributions are often found, therefore radius distribution needs to be modeled appropriately for cylindrical DDSs [37].

In this work we expand the previously developed mathematical approach to describing the diffusional drug release from spherical particles of distributed diameters and with evenly distributed drug, and conduct comparative analyses of the dimension distribution influence on drug release from DDSs of flat, cylindrical and spherical geometries. User friendly software was developed and is available as a supplement to help mathematical modeling and fitting experimental data of drug release from DDSs of spherical, cylindrical and flat geometries with distributed dimensions. 


\section{Model development}

\subsection{Drug release from DDSs of various geometries}

For model development, the following approximations and limitations were adopted:

- the drug is evenly distributed throughout the volume of the polymer matrix in the molecular form;

- the model considers only the initial stage of drug release, during which the process of drug release is controlled mainly by diffusion. It is assumed that the dissolution and degradation of the polymer happens at significantly later stages;

- the size and shape of the particles at the initial stage of drug release remains constant;

- the drug released only from amorphous phase.

Thus, in this work it is assumed that the release of the drug from the polymeric DDSs occurs mostly by the diffusion mechanism. This is generally the case at early stage of drug release from most DDSs [17]. The diffusion process is described by the Fick's second law and has the following forms:

$$
\begin{aligned}
& \frac{\partial C}{\partial t}=D \frac{\partial^{2} C}{\partial a^{2}}, \\
& \frac{\partial C}{\partial t}=D\left(\frac{\partial^{2} C}{\partial a^{2}}+\frac{1 \partial C}{a \partial a}\right), \\
& \frac{\partial C}{\partial t}=D\left(\frac{\partial^{2} C}{\partial a^{2}}+\frac{2 \partial C}{a \partial a}\right),
\end{aligned}
$$

for the flat (membrane), cylindrical and spherical DDSs, respectively, where $C$ is the drug concentration, $D$ is the diffusion coefficient, and $a$ describes spatial position in the membrane, cylinder or sphere depending on the DDS geometry. The drug is assumed to be uniformly distributed in the DDS with the initial concentration $\left(C_{0}\right)$ at $t=0$, therefore the initial condition in all cases is:

$$
C(a, 0)=C_{0} \text {. }
$$

The boundary conditions can be represented as:

$$
\begin{aligned}
& C(0, t)=C(H, t)=0, \\
& \left\{\begin{array}{l}
C(0, t) \leq Z \\
C(R, t)=0
\end{array}\right.
\end{aligned}
$$

for the membrane, and cylinder or sphere respectively, where $H$ is the membrane thickness, $R$ is the cylinder or sphere radius, $Z$ is a finite number.

Eqs. (1) with initial and boundary conditions (2-3) can be readily solved by using Laplace transform [38]. A more detailed solution for a cylindrical DDS is presented in [37]. Using a similar approach, amount of drug released from the spherical and flat DDSs with uniform distribution can be found, yielding for the amount of drug released from the DDSs:

$$
\begin{aligned}
& \hat{Q}(s)=\frac{2 C_{0} H A}{s \sqrt{s t_{d}}}\left[\frac{\cosh \sqrt{s t_{d}}-1}{\sinh \sqrt{s t_{d}}}\right]=M \frac{2}{s \sqrt{s t_{d}}}\left[\frac{\cosh \sqrt{s t_{d}}-1}{\sinh \sqrt{s t_{d}}}\right], \\
& \hat{Q}(s)=\frac{2 \pi L R^{2} C_{0}}{s \sqrt{s t_{d}}}\left[\frac{I_{1}\left(\sqrt{s t_{d}}\right)}{I_{0}\left(\sqrt{s t_{d}}\right)}\right]=M \frac{2}{s \sqrt{s t_{d}}}\left[\frac{I_{1}\left(\sqrt{s t_{d}}\right)}{I_{0}\left(\sqrt{s t_{d}}\right)}\right], \\
& \hat{Q}(s)=\frac{4 \pi R^{3} C_{0}}{s \sqrt{s t_{d}}}\left[\frac{1}{\tanh \sqrt{s t_{d}}}-\frac{1}{\sqrt{s t_{d}}}\right]=M \frac{3}{s \sqrt{s t_{d}}}\left[\frac{1}{\tanh \sqrt{s t_{d}}}-\frac{1}{\sqrt{s t_{d}}}\right],
\end{aligned}
$$

for membrane, cylinder and sphere respectively. Where $L$ is the length of the fiber, $A$ is the membrane area, $M$ is the amount of drug loaded into the DDS and $I_{1}$ and $I_{0}$ are the modified Bessel functions of the first kind, zero and first order, respectively. Further 


$$
t_{d}=\frac{a^{2}}{D}
$$

is the characteristic time of diffusion, where $a$ here stands for $H$ or $R$.

\subsection{Modelling the size distribution}

In order to take into account the distribution in dimensions, the morphology of a DDS should be studied and the dimensions of a representative ensemble of element of DDS should be measured, for example, using the ImageJ software (National Institutes of Health, MD, USA) as previously described [37]. The results of this analysis are usually presented as a histogram so that the probability $\left(p\left(a_{\mathrm{i}}\right)\right)$ of $i$-th group with dimensions (thickness or radius) in the range between $a_{\mathrm{i}}$ and $a_{\mathrm{i}+1}\left(a_{\mathrm{i}} \leq a \leq a_{\mathrm{i}+1}\right)$, can be calculated as:

$$
p\left(a_{i}\right)=\frac{n\left(a_{i}\right)}{n_{\text {total }}},
$$

where $n\left(a_{\mathrm{i}}\right)$ is the number of elements with the dimensions in the range and $n_{\text {total }}$ is the total number of all elements. For example of such histogram see inserts in the Fig. 6 below.

In order to quantify the dispersion of the size distribution the coefficient of variation $(C V)$ can be used:

$$
C V=\frac{s_{N}}{a_{m}},
$$

Where $a_{\mathrm{m}}$ is the mean dimension of all DDS elements and $s_{N}$ is a standard deviation of a discrete random variable $\left(s_{N}=\sqrt{\sum_{i=1}^{n}\left(a_{i}-a_{m}\right)^{2} p\left(a_{i}\right)}\right)$. This coefficient was used as a measure for the extent of the distribution for both the experimental and simulated data.

The approach for taking into account the distribution in dimensions was previously presented for flat and cylindrical systems [36], [37]. The course of the model development for spheres is very similar. In particular, the amount of drug $M_{i}=V_{i} C_{0}$ (where $V_{i}$ is the volume of the i-th group) contained in i-th group of DDS can be represented as:

$$
\begin{aligned}
M_{i} & =A_{i} a_{i} C_{0}, \\
M_{i} & =L_{i} \pi a_{i}^{2} C_{0}, \\
M_{i} & =\frac{4}{3} \pi a_{i}^{3} C_{0},
\end{aligned}
$$

for membrane, cylinder and sphere respectively, where $A_{i}$ is the membrane area and $L_{i}$ is the length of fibers of the $\mathrm{i}$-th group. Therefore, the total amount of drug contained in the whole $\operatorname{DDS}\left(M_{\text {total }}\right)$ is:

$$
M_{\text {total }}=\sum_{i=1}^{n} M_{i}
$$

This equation can be rewritten in terms of an effective average dimension of DDS $(\bar{a})$ for different geometries as:

$$
\bar{a}=\sqrt[q]{\sum_{i=1}^{n} a_{i}^{q} p\left(a_{i}\right)},
$$

where $q$ is 1,2 and 3 for the membrane, cylinder and sphere respectively. The amount of drug contained in the each DDS can then be determined as:

$$
M_{i}=M_{\text {total }}\left(\frac{a_{i}}{\bar{a}}\right)^{q} p\left(a_{i}\right) \text {. }
$$


Adding the amount of drug released from each group with dimension $a_{i}$ of the DDS (using $a_{i}$ instead of $a$ in Eqs (4)) the amount of drug released from the whole DDS at the time $t$ can be found as:

$$
\begin{gathered}
\hat{Q}(s)_{\text {total }}=M_{\text {total }} \sum_{i=1}^{n} \frac{a_{i}}{\bar{a}} p\left(a_{i}\right) \frac{2}{s \sqrt{s t_{d i}}}\left[\frac{\cosh \sqrt{s t_{d i}}-1}{\sinh \sqrt{s t_{d i}}}\right]+\frac{Q_{0}}{s}, \\
\hat{Q}(s)_{\text {total }}=M_{\text {total }} \sum_{i=1}^{n}\left(\frac{a_{i}}{\bar{a}}\right)^{2} p\left(a_{i}\right) \frac{2 I_{1}\left(\sqrt{s t_{d i}}\right)}{s \sqrt{s t_{d i}}\left(\sqrt{s t_{d i}}\right)}+\frac{Q_{0}}{s}, \\
\hat{Q}(s)_{\text {total }}=M_{\text {total }} \sum_{i=1}^{n}\left(\frac{a_{i}}{\bar{a}}\right)^{3} p\left(a_{i}\right) \frac{3}{s \sqrt{s t_{d i}}}\left[\operatorname{ctanh} \sqrt{s t_{d i}}-\frac{1}{\sqrt{s t_{d i}}}\right]+\frac{Q_{0}}{s},
\end{gathered}
$$

for the membrane, cylindrical and spherical DDS respectively, where $t_{\mathrm{di}}$ is the characteristic time of diffusion for the dimension $a_{i}$. Further, $Q_{0}$ is the amount of drug present on the surface of elements of a DDS and released immediately, that needs to be taken into account in many cases [36], [37].

As an example of a size distribution the normal distribution was considered. To be used in Eqs. 12, the distribution was discretized [29], so that:

$$
p\left(a_{i}\right)=\beta \exp \left[-\frac{\left(a_{i}-a_{m}\right)^{2}}{2 \sigma^{2}}\right]
$$

where $\beta=1 / \sum_{i=1}^{n} \exp \left[-\frac{\left(a_{i}-a_{m}\right)^{2}}{2 \sigma^{2}}\right], \sigma$ is the standard deviation of the random variable.

\subsection{Numerical analysis}

The numerical inverse Laplace transform was performed using the custom-written program in Python programming language implemented in Enthought Canopy package. The numerical Laplace transform was calculated using the Talbot algorithm [39]. An equal weighting was used for nonlinear regressions. The user-friendly software based on the size distribution model for DDSs of all geometries is provided as supplementary files. It allows to fit experimental data determining the diffusion coefficient and to predict the theoretical drug release profile when the diffusion coefficient is given. The installation instructions as well as the example files of particles radius distribution and experimental release profile based on the data from [40] are also given in the supplementary files.

\section{Results and discussion}

\subsection{Effect of the initial parameters on the drug release profile}

First, the effect of the model parameters, such as $C V$ value and the diffusion coefficient, on the drug release profile from various geometries DDSs was investigated. In order to estimate the dependence of the drug release time on the elements size distribution a number of distributions with different $C V$ values in comparison to the homogeneous model $(C V=0)$ was used to fit the quasi-experimental data. The results of modeling, the distributions as well as the time of release $90 \%$ of the drug $\left(t_{90 \%}\right)$ for each distribution at $t_{\mathrm{d}}=250 \mathrm{~h}$ (which corresponds to $D=2.8 \cdot 10^{-17}$ $\mathrm{cm}^{2} / \mathrm{s}$ for membrane, cylindrical and spherical DDSs are presented in Fig. 1. For the homogeneous model, the value of the elements dimension (membrane thickness or fiber/sphere 
radius) was assumed to be equal to the mean value $a_{\text {mean }}=50 \mathrm{~nm}$. In the experimental papers this value is often reported. In addition to the homogeneous distribution, normal distributions with the same mean value, but with different standard deviations and therefore $C V$ values $(C V=0.15$; 0.30 and 0.45 ), were considered as well as the two-modal normal distribution with $C V=0.45$.

In case of membranes the difference of drug release profiles obtained by the size distribution and homogeneous models is relatively small (Fig. 1a). There are no significant differences in the release profiles with different $C V$ before at least $60 \%$ of drug is released. The times of $90 \%$ drug released $\left(t_{90 \%}\right)$ for different distributions are presented in Table 1 . The value of $t_{90 \%}$ is only $16 \%$ larger if the $C V$ is 0.45 compared to the homogeneous model. This value also rises for the twomodal distribution. The difference in drug release profiles is much bigger for cylindrical DDSs, as it was described in the previous article [37]. It can be seen from the Fig. 1b and the Table 1, that with increasing of $C V$ value from 0 to $0.45, t_{90 \%}$ almost doubled. The results for two-modal distribution are similar to the one-modal one with the same $C V$ value. Significant differences in the profiles are not observed until about $25 \%$ of released drug. Similarly, in the spherical DDSs there are no significant differences in the profiles of drug releases at the initial stage (approximately before $20 \%$ of drug released), as it is shown in Fig. 1c. Then the differences become more noticeable and the release times of $90 \%$ of the drug from the DDS for different distributions may differ several times. The times of $90 \%$ drug released for each distribution as well as relative difference between $t_{90 \%}$ values determined by using the size distribution and homogeneous models are also shown in the Table 1.

It is seen, that as the value of $C V$ increases, the difference in the values of $t_{90 \%}$ also increases according to a quadratic law. So, $t_{90 \%}$ value rise from $45 \mathrm{~h}$ in case of homogeneous model $(C V=0)$ to $158 \mathrm{~h}$ for size distribution model with $C V=0.45$ that is more than three times. Time of release $90 \%$ of drug for two-modal distribution turned out to be close in value to $t_{90 \%}$ for a unimodal distribution with the smallest $C V$ value. This confirms that the accuracy of the model is affected not only by the value of $C V$, but also by the form of the distribution, as it was noted in the previous paper [37].

(a)

(b)

(c)

Fig. 1. Drug release profiles for (a) membrane, (b) fiber and (c) sphere geometry DDSs for homogeneous models (solid lines) and distributed models with CV values of $0.15,0.30$ and 0.45 represented by dash-doted dashed and short dashed lines respectively. The insert shows size distributions used.

In order to assess particle size distribution influence on the determination of the diffusion coefficient the quasi-experimental profiles of the drug release were simulated using size distribution model with different $C V$ values for each geometrical form of DDS. To simulate experimental data the diffusion coefficient was assumed to be $D_{\text {assumed }}=2.8 \cdot 10^{-14} \mathrm{~cm}^{2} / \mathrm{s}$, and the mean radius was $50 \mathrm{~nm}$. To model a realistic experimental profile, a random $5 \%$ error was added 
to each point of quasi-experimental data. The results of fitting this data by homogeneous and size distribution models with a value of $C V=0.30$ are shown in Fig. 2. Coefficients of diffusion were determined in each case and are shown in the Table 2. Thus, the error in determining of diffusion coefficient in case of spherical size distribution model is approximately three times more than in the case of cylindrical model. It indicates that accounting for the distribution of elements by size is more important in case of polymer micro- and nanoparticles then for fibrous scaffolds as a drug carrier. Withal the difference in time of $90 \%$ drug release was less significant: about two times for spherical and about 1.6 times for cylindrical DDSs when using the different models.

Also, as can be seen from the Fig.2, both homogeneous and size distribution models yield very good fitting for all geometrical forms. Thus, the quality of fitting of the experimental data alone cannot be used to justify the use of the homogeneous model. The use of the simpler model can lead to incorrect parameter definition. Therefore, it is necessary to take into account size distribution even in cases when curves produce similar quality fitting.

a)

Fig. 2. The drug release profiles based on quasi-experimental data for membrane (a), cylindrical (b) and spherical (c) DDSs. The fitting was performed by the homogeneous (dashed lines) and size distribution (solid lines) models.

The relative errors in determining of diffusion coefficient when using the homogeneous $\left(D_{h}\right)$ versus size distribution $\left(D_{d}\right)$ model for various $C V$ value $(0.05,0.1,0.2,0.3$ and 0.4$)$ for all forms of DDSs was calculated by Eq. (12) and presented in Fig. 3.

$$
\text { Error }=\frac{D_{d}-D_{h}}{D_{d}}
$$

Fig. 3. Relative errors in determining diffusion coefficient when using the models with different size distributions with various $C V$ values for membrane (triangles), fiber (diamonds) and spherical (circles) DDSs.

Fig. 3 shows that the relative error in determining the diffusion coefficient increases with rising of $C V$ value. The smallest errors are for DDS with membrane geometry, where it is possible to use simpler homogeneous model for all $\mathrm{CV}$ values considered. The relative error in determining of diffusion coefficient for cylindrical DDSs increases 3 times compared to the membrane geometry. In this case it is necessary to take into account the fiber radius distribution, as was previously described [37]. The relative error for the spherical systems is the largest, about two times greater than for cylindrical DDSs. It can be seen from the Fig. 3 that the use of simpler homogeneous model for release profiles from DDS with size distribution leads to less than $10 \%$ error in the determined diffusion coefficient when $C V$ values are less than $0.40,0.20$ and 0.10 for membrane, cylinder and sphere respectively, and is therefore acceptable.

The increase in the influence of size distribution for spherical DDSs can be explained by the fact that while the volume of the cylinder is proportional to the square of its radius the volume of the sphere is proportional to the cube of radius. Therefore distribution of spheres over radii affects the drug release more significantly than in the case of a cylindrical system, which leads to a greater error when simpler homogeneous model is used for the sphere geometries. 


\subsection{Fitting the experimental data}

In order to demonstrate the developed model, the experimental data of drug release from spherical DDSs were used [41]. The effect of copolymer composition on drug release profile for progesterone-loaded poly(lactic-co-glycolic acid) (PLGA) particles was investigated [41]. To test the developed model, the 50:50 DL-lactide/glycolide copolymers with polymer/drug ratios of 10:1 and 20:1 (10 wt.\% and 5 wt.\% of progesterone), designated as F1 and F3, respectively, were chosen. These copolymers meet the basic requirements of our model. In particular, particles of such copolymers for the most part have a spherical shape, as has been shown on SEM images. According to the images, the particle size distribution was calculated by a method similar to the method described in this paper. It was also noted that for these composites the time of degradation is long enough and does not need to be taken into account for the initial release stage [41]. In addition, it was suggested, based on the data obtained, that the drug was dissolved in the polymer in molecular form. As the progesterone is a small hydrophobic drug, it diffused predominantly through the polymer rother than through the water filled pores. Thus, it was stated that during the first few hours the release of the drug is controlled by the diffusion of the drug molecules through the polymer matrix [41]. In Fig. 4 experimental results of progesterone release from F1 and F3 copolymers and their particle size distribution are presented. These data were fitted using the developed size distribution model (solid line in Fig 4) and the homogeneous model (dashed line in Fig 4).

a)

b)

Fig. 4. Simulated progesterone release profiles for F1 (a) and F3 composites (b) based on the experimental data [41]; the dashed and solid lines represent fitting with the homogeneous and the size distribution models respectively. The corresponding sphere radius distributions from [41] are in the inserts.

It was noted in the article [41] that progesterone is not completely released from the systems. The amounts of drug released after the $168 \mathrm{~h}$ of observation where only 43 and $83 \%$ for F1 and F3 systems, respectively. At the same time the results of Fourier transform infrared spectroscopy showed, that there were no chemical interactions between polymer and progesterone. In this regard, it can be hypothesized that the irreversibly bound fraction of drug was due to the incomplete wetting of particles due to their low swelling ability. On the other hand, it is seen from the experimental data from [41] that the profiles of drug release for all systems continue to rise after $168 \mathrm{~h}$ of observation. Thus, it is possible that the release of $100 \%$ of progesterone can be achieved over longer observation times.

It can be seen in Fig.4, that release profiles obtained using different models are quite close, which is partly due to relatively small $C V$ values for these composites $(0.17$ for F1 and 0.23 for F3). According to the fitting results, the diffusion coefficients of the drug in the copolymer were calculated and presented in Table 3. In [41] the drug release was fitted using the diffusion model developed in [42], and determined diffusion coefficients are also presented in Table 3. Besides, it was mentioned in [41] that the diffusion coefficient of a drug in a similar system was determined based on the previously published data [43] and amounted to $2.1 \times 10^{-17} \mathrm{~m}^{2} / \mathrm{s}$. As can be seen in Table 3, when using the developed model, diffusion coefficients of the same order of magnitude were obtained for F1. At the same time the values in [41] are twice smaller for F3 composite. 
The relative error in determining of diffusion coefficient using the size distribution versus homogeneous model is about $68 \%$ for $\mathrm{F} 1$ composite and about $26 \%$ for $\mathrm{F} 3$. The second error corresponds well to the previously presented relative error graph (Fig.3). The error for F1 is about $10 \%$ higher than expected, which can be due to a large experimental error. All obtained diffusion coefficients no more than twice higher than values calculated in [41] due to the difference in the mathematical approaches used. Besides, in developed size distribution model the amount of drug presents on the surface of particles $\left(Q_{0}\right)$ was used, which may cause an increase in the accuracy of calculations.

Thus, the developed size distribution diffusion model was verified by using the published experimental data. The DDS used in the experiment satisfied the requirements of the model. The results demonstrated that the developed model adequately describes the experimental results and allows defining diffusion coefficients with better accuracy, as the size distribution is appropriately modelled. The difference in the determined diffusion coefficient when using the homogeneous vs the size distribution model is significant even for relatively small $C V$ values of the particle size distribution for spherical particles.

\section{Conclusion}

Mathematical model for diffusional drug release from DDSs of various geometries taking into account the distribution in dimensions was presented. Validation of the model for spherical geometry DDS was performed using published experimental data. It was confirmed that the size distribution significantly influence the shape of release profile and the time of drug release for fibers and spherical particles, but not for membrane geometry DDSs. The relative error in determining of diffusion coefficient was shown to be less than $10 \%$ for the membranes, but increases significantly for cylindrical and spherical DDSs. Release profiles from spherical DDSs demonstrate much higher sensitivity to size distribution then fiber based DDSs. At the same time the fitting curves of experimental results for homogeneous and size distribution models are visually close to each other, which could lead to difficulties in realizing that incorrect model is used, when not taking size distribution into account. Thus, the need to take into account the particle size distribution when modelling drug release is obvious for fibers and especially significant for micro- and nanoparticles. The obtained results can be used to determine the geometry of DDS to achieve the desired therapeutic effect by controlled release of the drug.

The developed modes were implemented in the user-friendly software using Python programming language. The software with instruction and test files are provided as supplementary files.

\section{Acknowledgements}

This work was financially supported by the Ministry of Science and Higher Education of the Russian Federation, Federal Target Program (agreement \#14.575.21.0140, unique identifier RFMEFI57517X0140). 


\section{References}

[1] A. Srivastava, T. Yadav, S. Sharma, A. Nayak, A. Akanksha Kumari, and N. Mishra, "Polymers in Drug Delivery," J. Biosci. Med., vol. 04, no. 01, pp. 69-84, 2016.

[2] B. Karolewicz, "A review of polymers as multifunctional excipients in drug dosage form technology," Saudi Pharm. J., vol. 24, no. 5, pp. 525-536, 2016.

[3] A. S. H. Makhlouf, 1 - Historical development of drug delivery systems: From conventional macroscale to controlled, targeted, and responsive nanoscale systems. Elsevier Ltd., 2018.

[4] P. Khatri, D. Desai, N. Shelke, and T. Minko, "Role of plasticizer in membrane coated extended release oral drug delivery system," J. Drug Deliv. Sci. Technol., vol. 44, pp. 231-243, Apr. 2018.

[5] J.-J. Li, Y.-Y. Yang, D.-G. Yu, Q. Du, and X.-L. Yang, "Fast dissolving drug delivery membrane based on the ultra-thin shell of electrospun core-shell nanofibers," Eur. J. Pharm. Sci., vol. 122, pp. 195-204, Sep. 2018.

[6] V. L. Kudryavtseva, L. Zhao, S. I. Tverdokhlebov, and G. B. Sukhorukov, "Fabrication of PLA/CaCO3 hybrid micro-particles as carriers for water-soluble bioactive molecules," Colloids Surfaces B Biointerfaces, vol. 157, pp. 481-489, Sep. 2017.

[7] A. D. Badaraev, A. L. Nemoykina, E. N. Bolbasov, and S. I. Tverdokhlebov, "PLLA scaffold modification using magnetron sputtering of the copper target to provide antibacterial properties," Resour. Technol., vol. 3, no. 2, pp. 204-211, Jun. 2017.

[8] R. K. Saini, L. P. Bagri, A. K. Bajpai, and A. Mishra, 11 -Responsive polymer nanoparticles for drug delivery applications. Elsevier Ltd., 2018.

[9] B. Bahrami, M. Hojjat-farsangi, H. Mohammadi, and E. Anvari, "Nanoparticles and targeted drug delivery in cancer therapy," vol. 190, no. April, pp. 64-83, 2017.

[10] P. Malyala and D. T. O'Hagan, Polymeric Particles as Vaccine Delivery Systems. Elsevier Ltd, 2016.

[11] B. Kumar, K. Jalodia, P. Kumar, and H. K. Gautam, "Recent advances in nanoparticlemediated drug delivery," J. Drug Deliv. Sci. Technol., vol. 41, pp. 260-268, 2017.

[12] M. H. Xiong, Y. Bao, X. Z. Yang, Y. H. Zhu, and J. Wang, "Delivery of antibiotics with polymeric particles," Adv. Drug Deliv. Rev., vol. 78, pp. 63-76, 2014.

[13] D. Klose, F. Siepmann, K. Elkharraz, and J. Siepmann, "PLGA-based drug delivery systems: Importance of the type of drug and device geometry," Int. J. Pharm., vol. 354, no. 1-2, pp. 95-103, 2008.

[14] N. S. Berchane, K. H. Carson, A. C. Rice-Ficht, and M. J. Andrews, "Effect of mean diameter and polydispersity of PLG microspheres on drug release: Experiment and theory," Int. J. Pharm., vol. 337, no. 1-2, pp. 118-126, 2007.

[15] N. A. Peppas and B. Narasimhan, "Mathematical models in drug delivery: How modeling has shaped the way we design new drug delivery systems," J. Control. Release, vol. 190, pp. 75-81, 2014.

[16] C. Busatto, J. Pesoa, I. Helbling, J. Luna, and D. Estenoz, "Effect of particle size, polydispersity and polymer degradation on progesterone release from PLGA microparticles: Experimental and mathematical modeling," Int. J. Pharm., vol. 536, no. 1, pp. 360-369, Jan. 2018.

[17] J. Siepmann and F. Siepmann, "Modeling of diffusion controlled drug delivery," $J$. Control. Release, vol. 161, no. 2, pp. 351-362, 2012.

[18] J. Siepmann, F. Lecomte, and R. Bodmeier, "Diffusion-controlled drug delivery systems: Calculation of the required composition to achieve desired release profiles," J. Control. Release, vol. 60, no. 2-3, pp. 379-389, 1999.

[19] J. Liang, B. Yang, and J. Deng, "Polylactide-based chiral particles with enantiodifferentiating release ability," Chem. Eng. J., vol. 344, no. January, pp. 262-269, 2018.

[20] A. Chebil, M. Léonard, J. L. Six, C. Nouvel, and A. Durand, "Nanoparticulate delivery 
systems for alkyl gallates: Influence of the elaboration process on particle characteristics, drug encapsulation and in-vitro release," Colloids Surfaces B Biointerfaces, vol. 162, pp. 351-361, 2018.

[21] C. W. Shields et al., "Encapsulation and controlled release of retinol from silicone particles for topical delivery," J. Control. Release, vol. 278, no. March, pp. 37-48, 2018.

[22] C. Busatto, J. Pesoa, I. Helbling, J. Luna, and D. Estenoz, "Effect of particle size, polydispersity and polymer degradation on progesterone release from PLGA microparticles: Experimental and mathematical modeling," Int. J. Pharm., vol. 536, no. 1, pp. 360-369, 2018.

[23] F. Ungaro et al., "Microparticle-embedded fibroin/alginate beads for prolonged local release of simvastatin hydroxyacid to mesenchymal stem cells," Carbohydr. Polym., vol. 175, no. July, pp. 645-653, 2017.

[24] L. Pastorino, E. Dellacasa, P. Petrini, and O. Monticelli, "Stereocomplex poly(lactic acid) nanocoated chitosan microparticles for the sustained release of hydrophilic drugs," Mater. Sci. Eng. C, vol. 76, pp. 1129-1135, 2017.

[25] S. Liu et al., "Use of asymmetric multilayer polylactide nanofiber mats in controlled release of drugs and prevention of liver cancer recurrence after surgery in mice," Nanomedicine Nanotechnology, Biol. Med., vol. 11, no. 5, pp. 1047-1056, 2015.

[26] T. Dappert and C. Thies, "Statistical models for controlled release microcapsules: rationale and theory," J. Memb. Sci., vol. 4, no. C, pp. 99-113, 1978.

[27] A. Hoffman, M. Donbrow, S. T. Gross, S. Benita, and R. Bahat, "Fundamentals of release mechanism interpretation in multiparticulate systems: determination of substrate release from single microcapsules and relation between individual and ensemble release kinetics," Int. J. Pharm., vol. 29, no. 2-3, pp. 195-211, 1986.

[28] M. Donbrow, A. Hoffman, and S. Benita, "Variation of Population Release Kinetics in Polydisperse Multiparticulate Systems (Microcapsules, Microspheres, Droplets, Cells) with Heterogeneity of One, Two or Three Parameters in the Population of Individuals," J. Pharm. Pharmacol., pp. 93-96, 1988.

[29] M. Grassi, I. Colombo, and R. Lapasin, "Drug release from an ensemble of swellable crosslinked polymer particles," J. Control. Release, vol. 68, no. 1, pp. 97-113, 2000.

[30] F. E. Eichie and R. S. Okor, "Modelling of drug release from ensembles of aspirin microcapsules of certain particle size distribution," Trop. Jounal Pharm. Res., vol. 2, no. 1, pp. 137-145, 2003.

[31] E. Kaunisto, M. Marucci, P. Borgquist, and A. Axelsson, "Mechanistic modelling of drug release from polymer-coated and swelling and dissolving polymer matrix systems," Int. J. Pharm., vol. 418, no. 1, pp. 54-77, 2011.

[32] N. Coceani, L. Magarotto, D. Ceschia, I. Colombo, and M. Grassi, "Theoretical and experimental analysis of drug release from an ensemble of polymeric particles containing amorphous and nano-crystalline drug," Chem. Eng. Sci., vol. 71, pp. 345-355, 2012.

[33] D. Caccavo, G. Lamberti, M. M. Cafaro, A. A. Barba, J. Kazlauske, and A. Larsson, "Mathematical modeling of the drug release from an ensemble of coated pellets," Br. J. Pharmacol., vol. 174, no. 12, pp. 1797-1809, 2017.

[34] B. Hong et al., "Effect of tetrodotoxin pellets in a rat model of postherpetic neuralgia," Mar. Drugs, vol. 16, no. 6, pp. 1-14, 2018.

[35] T. J. Sill and H. A. von Recum, "Electrospinning: Applications in drug delivery and tissue engineering," Biomaterials, vol. 29, no. 13, pp. 1989-2006, 2008.

[36] D. G. Petlin, M. Rybachuk, and Y. G. Anissimov, "Pathway Distribution Model for Solute Transport in Stratum Corneum," J. Pharm. Sci., vol. 104, no. 12, pp. 4443-4447, 2015.

[37] D. G. Petlin, A. A. Amarah, S. I. Tverdokhlebov, and Y. G. Anissimov, "A fiber distribution model for predicting drug release rates," J. Control. Release, vol. 258, pp. 218-225, 2017.

[38] E. J. Carr and G. Pontrelli, "Modelling mass diffusion for a multi-layer sphere immersed 
in a semi-infinite medium: application to drug delivery," Mathematical Biosciences, no. April, 2018.

[39] A. Talbot, "The accurate numerical inversion of laplace transforms," IMA J. Appl. Math. (Institute Math. Its Appl., vol. 23, no. 1, pp. 97-120, 1979.

[40] M. Dhanka, C. Shetty, and R. Srivastava, "Methotrexate loaded gellan gum microparticles for drug delivery," Int. J. Biol. Macromol., vol. 110, pp. 346-356, 2018.

[41] Y. Zhang, T. Shams, A. H. Harker, M. Parhizkar, and M. Edirisinghe, "Effect of copolymer composition on particle morphology and release behavior in vitro using progesterone," Mater. Des., vol. 159, pp. 57-67, 2018.

[42] M. Eltayeb, E. Stride, M. Edirisinghe, and A. Harker, "Electrosprayed nanoparticle delivery system for controlled release,” Mater. Sci. Eng. C, vol. 66, pp. 138-146, Sep. 2016.

[43] G. Acharya et al., "A study of drug release from homogeneous PLGA microstructures," J. Control. Release, vol. 146, no. 2, pp. 201-206, 2010. 

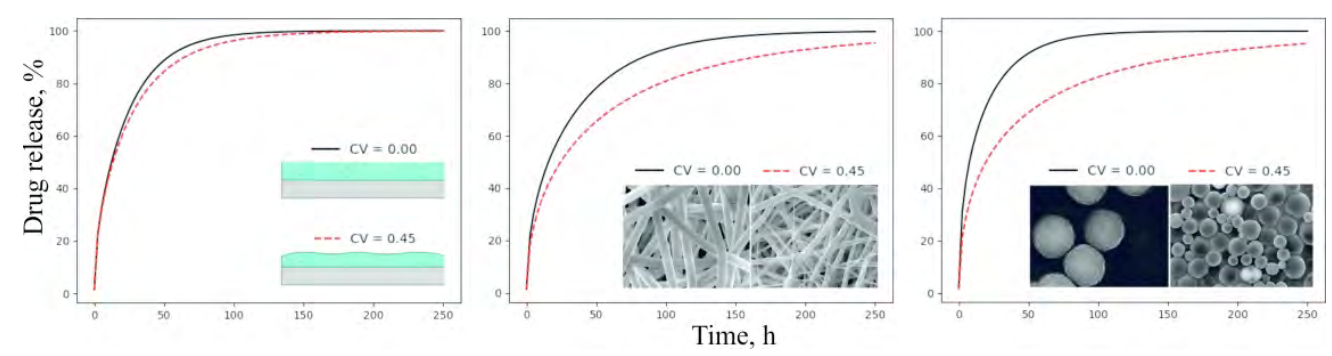

Graphical abstract 
Table 1. Times of $90 \%$ drug released for different size distributions

\begin{tabular}{c|ccc} 
& \multicolumn{3}{|c}{$t_{90 \%,}, \mathrm{~h}$} \\
\hline CV value & membrane & cylinder & sphere \\
\hline homogeneous, 0 & 55 & 83 & 45 \\
0.15 & 56 & 90 & 58 \\
0.30 & 58 & 120 & 96 \\
0.45 & 64 & 162 & 158 \\
double-peak 0.45 & 67 & 162 & 138 \\
\hline
\end{tabular}


Table 2. The diffusion coefficients for different DDSs when using the homogeneous versus size distribution models

\begin{tabular}{c|ccc} 
& \multicolumn{3}{|c}{ Diffusion coefficient, $\cdot 10^{-14} \mathrm{~cm}^{2} / \mathrm{s}$} \\
\hline CV value & membrane & cylinder & sphere \\
homogeneous, 0 & 2.8 & 2.2 & 1.5 \\
0.30 & 2.6 & 3.0 & 3.1 \\
\hline
\end{tabular}


Table 3. The progesterone diffusion coefficients calculated by using the different models in comparison to results obtained in [41]

\begin{tabular}{c|c|ccc}
\hline \multirow{2}{*}{ Composite } & \multirow{2}{*}{ CV } & \multicolumn{3}{|c}{ Diffusion coefficient, $10^{-17} \mathrm{~m}^{2} / \mathrm{s}$} \\
\cline { 3 - 5 } & & $\begin{array}{c}\text { Homogeneous } \\
\text { model }\end{array}$ & $\begin{array}{c}\text { Size distribution } \\
\text { model }\end{array}$ & Ref. [41] \\
\hline F1 & 0.17 & 2.53 & 1.51 & $1.40 \pm 0.40$ \\
F3 & 0.23 & 0.87 & 1.18 & $0.68 \pm 0.09$ \\
\hline
\end{tabular}




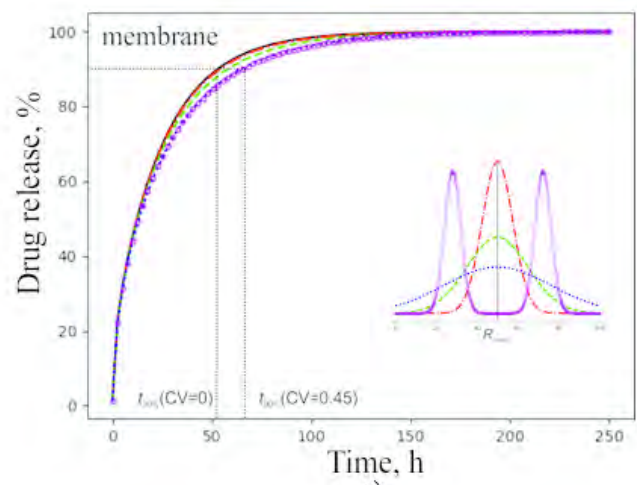

a)

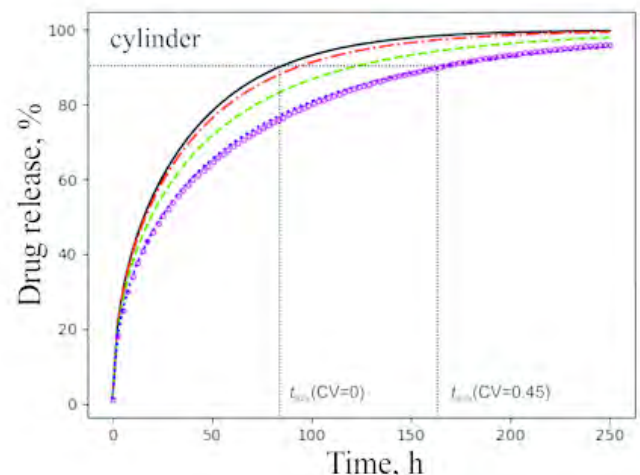

b)

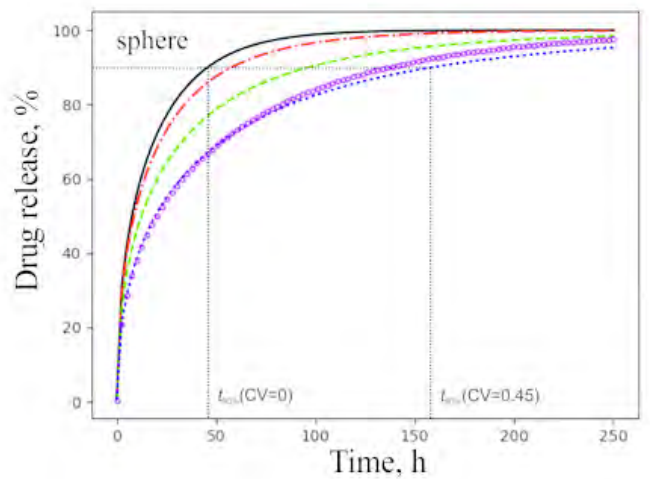

Fig. 1. Drug release profiles for (a) membrane, (b) fiber and (c) sphere geometry DDSs for homogeneous models (solid lines) and distributed models with CV values of $0.15,0.30$ and 0.45 represented by dashdoted dashed and short dashed lines respectively. The insert shows size distributions used. 


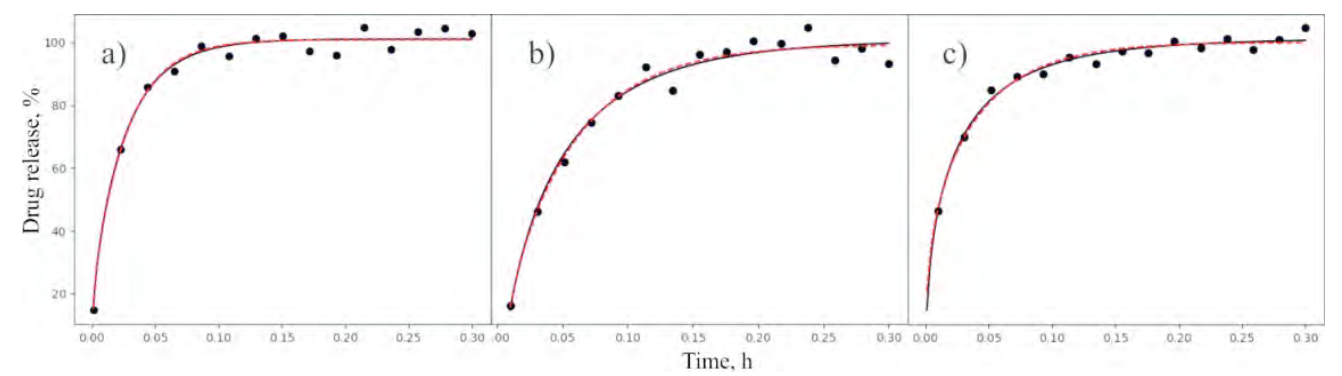

Fig. 2. The drug release profiles based on quasi-experimental data for membrane (a), cylindrical (b) and spherical (c) DDSs. The fitting was performed by the homogeneous (dashed lines) and size distribution (solid lines) models. 


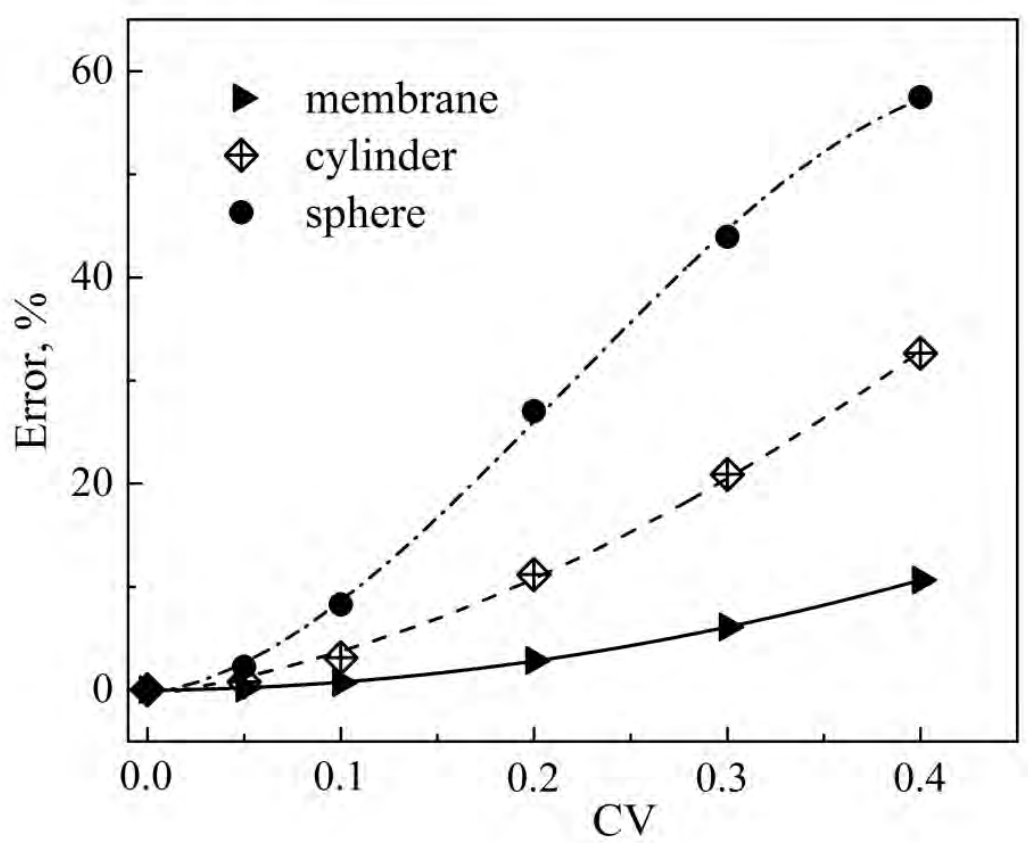

Fig. 3. Relative errors in determining diffusion coefficient when using the models with different size distributions with various CV values for membrane (triangles), fiber (diamonds) and spherical (circles) DDSs.

$279 \times 215 \mathrm{~mm}(300 \times 300 \mathrm{DPI})$ 

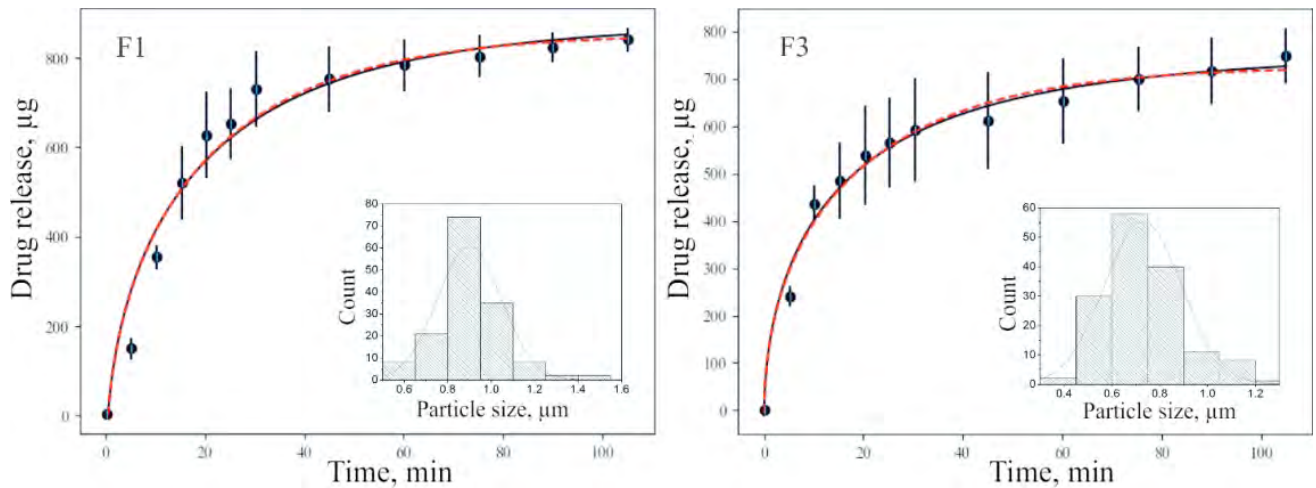

Fig. 4. Simulated progesterone release profiles for $F 1$ (a) and F3 composites (b) based on the experimental data [41]; the dashed and solid lines represent fitting with the homogeneous and the size distribution models respectively. The corresponding sphere radius distributions from [41] are in the inserts. 


$\begin{array}{ll}3.19885 & 0.001 \\ 12.47934 & 18.56823 \\ 23.30657 & 25.28129 \\ 32.58706 & 31.3357 \\ 41.86755 & 38.02342 \\ 61.97527 & 50.13225 \\ 84.66091 & 54.86939 \\ 112.76016 & 61.55711 \\ 142.14837 & 67.07955 \\ 202.47154 & 71.56336 \\ 324.14905 & 76.3005 \\ 563.6372 & 77.33912 \\ 923.51389 & 81.29096 \\ 1443.99458 & 83.79886\end{array}$




\section{INSTRUCTION}

The easiest way:

1. Download the "Enthought Canopy" IDE from the https://www.enthought.com/product/canopy/ and install it.

2. Run the Enthought Canopy and click to the "Package Manager" icon.

3. Go to the tab "Installed" at the left and select the following packages: matplotlib, scipy, numpy, tk_headers.

4. If you can not find them, go to the tab "Available" at the left. If you find them, go to item 6 .

5. Find and install the following packages in the list: matplotlib, scipy, numpy, tk_headers.

6. Close the Package Manager and open the "Editor" icon.

7. Select the program file (Distribution models.py) from the computer or create new file and copy

text of the program.

8. Run the project.

9. Select the geometry of the system in the window that opens.

10. Enter other initial paremeters (additional information about each parameter when you click on the button with a question mark "?").

Test distribution and release data for each geometry, you can also find in the supplementary materials.

11. Press the "ENTER" button.

12. Wait for the results, it may take a few minutes.

13. Save the results, if necessary.

For advanced users:

1. Download python3 installer from the https://www.python.org/downloads/ and install it.

2. Run command prompt.

3. Go to the directory containing this program in prompt.

4. Type the "python" or "python3"

5. If you see the symbol ">>>", type the "pip install matplotlib"

6. Type the "pip install scipy"

7. If the installation completed successfully, type the name of the program, including the file extension .py For example, "Distribution_models.py"

8. Select the geometry of the system in the window that opens.

9. Enter the other initial paremeters (additional information about each parameter when you click on the button with a question mark "?").

Test distribution and release data for each geometry you can also find in the supplementary materials.

10. Press the "ENTER" button.

11. Wait for the results, it may take a few minutes. 
12. Save the results, if necessary.

You can also use any other python compiler you have. 